\title{
Der Wille zum Stil - Die ästhetische „Umwertung der Werte“ im Fin de Siècle
}

\author{
MARCUS OTTO
}

\section{Einleitung}

In seinem berühmten Nietzsche-Buch formuliert Gilles Deleuze gleich zu Beginn eindrücklich, Nietzsche habe überhaupt erst den Begriff des Wertes in die Philosophie eingeführt. ${ }^{1}$ Denn mit dem Theorem der Umwertung hat Nietzsche im Rahmen seiner Genealogie (der Moral) nicht nur das „Perspektivische in jeder Wertschätzung“2 aufgezeigt, sondern vor allem durch die historische Problematisierung von Wertsetzungen die jeweils herrschenden Werte als Ausdruck eines jeweiligen vornehmen oder niedrigen Willen zur Macht dekonstruiert. Für Nietzsche werden Werte dergestalt schließlich von einem vermeintlich ethischen Problem zu einer Frage des ästhetischen Geschmacks und vor allem des Stils. Der Name Nietzsche steht in diesem Zusammenhang mithin für eine ästhetische Dekonstruktion der Ethik.

Im folgenden wird das berüchtigte Postulat der „Umwertung aller Werte“, wie es Nietzsche als Untertitel seines angekündigten, aber niemals verfassten, vermeintlichen Hauptwerkes „Der Wille zur Macht“ vorsah, daraufhin gelesen, dass es effektiv nicht auf die viel beschworene „große Politik“ oder gar dialektisch auf Gesellschaftsund Kulturkritik zielte, sondern vor allem als dezidiert ästhetische Umwertung konventioneller ethischer Werte einen diskursiven Stil hervorbrachte, der das sogenannte Fin de Siècle prägte und weit darüber hinaus vor allem im Sinne zynischer Provokation entsprechende Praktiken ästhetischer Selbstinszenierung gegenüber der Logik ethischer Werte inspirierte. Der Stil wurde dabei zum privile-

1 Vgl. Gilles Deleuze: Nietzsche et la philosophie, Paris: Presses Universitaires de France 2003 (orig.1962), S.1.

2 Vgl. Friedrich Nietzsche: Menschliches, Allzumenschliches. Ein Buch für freie Geister, Leipzig 1878, Kritische Studienausgabe, Bd. 2, herausgegeben von Giorgio Colli und Mazzino Montinari, Berlin: de Gruyter 1993, S.20. 
gierten Medium der Selbstkonstitution, indem er im Fin de Siècle eine ästhetische Umwertung der Werte konstituierte. Allerdings erfolgte diese Umwertung bei aller zweifellosen Singularität Nietzsches nicht allein aus dessen spezifischem Denk-Stil heraus, sondern entfaltete sich effektiv, indem sie zugleich Inspiration und Resonanz in einem elaboriert ästhetischen Lebens-Stil fand, wie ihn insbesondere die literarisch stilisierte Figur des Dandys im 19. Jahrhundert verkörperte und schließlich im Fin de Siècle als ausgesprochener décadent gesellschaftlich prekär exponierte. Wie im folgenden deutlich werden wird, ist diese beobachtete Affinität zwischen Nietzsche und der Figur des Dandys hinsichtlich einer solchen ästhetischen Umwertung der Werte im Zeichen des Stils wohl gleichermaßen naheliegend wie überraschend, also letztlich paradox. Dieser Essay widmet sich daher im Folgenden der Frage, inwiefern der vor allem literarisch stilisierte Dandy ${ }^{3}$ genealogisch als Wegbereiter des ästhetischen Denk-Stils Nietzsches und jener berüchtigten „Umwertung der Werte" gelten kann.

\section{Das Dandytum als ethisch-ästhetische Technologie des Selbst: „Beau Brummell“}

Der Dandy des 19. Jahrhunderts ist sicherlich eine der prominentesten Figuren, wenn es um das Verhältnis zwischen Ethik und Ästhetik geht. So wurde der Dandy zunächst vor allem als Modegeck, der ausschließlich auf seine ästhetische Selbstinszenierung bedacht war, wahrgenommen. Indem der Dandy, wie paradigmatisch an der Figur George „Beau“ Brummells deutlich wird, sich selbst zu einem Kunstwerk stilisierte, spielte er offensichtlich Ästhetik gegen Ethik aus. Zugleich bildete allerdings das Ensemble derjenigen Praktiken, durch die er sich selbst umfassend ästhetisierte, unweigerlich eine eigene Ethik aus. Denn die distinguierte und elaborierte Art und Weise, in der Brummell durch sein modisches Erscheinungsbild, sein zynisch arrogantes Auftreten, seinen eloquenten und durch das Spiel mit ausgesprochenen Paradoxa verblüffenden Konversationsstil sowie seinen extravaganten Lebensstil überhaupt gesellschaftlich glänzte, erforderte unweigerlich eine geradezu asketische Selbstbeherrschung. Ja, die Ästhetisierung des Selbst bedingte für den Dandy die Formierung einer eigenen Ethik, und zwar in der Form einer ausgesprochenen Umkehrung der im 19. Jahrhundert vorherrschenden utilitaristischen Ethik der bürgerlichen Gesell-

3 Siehe ausführlich zur literarischen Selbststilisierung des Dandys in der diskursiven Strategie der Selbstbehauptung Fernand Hörner: Die Behauptung des Dandys. Eine Archäologie, Bielefeld: transcript 2008. 
schaft. Dieses ästhetisch-ethische Programm des Dandys erschöpfte sich allerdings keineswegs im Imperativ der Selbstbeherrschung, sondern bedingte darüber hinaus insbesondere auch die Machtausübung gegen andere, und zwar im Modus der ästhetischen Selbstinszenierung des Dandys vor einem davon faszinierten Publikum. Im Falle Brummells, also zur Regency-Zeit, bezog diese dem Dandy inhärente Machtausübung den monarchischen Hof und sogar den König explizit mit ein. Denn der Status- und Amtsmacht des Königs, die durchaus institutionelle Autorität und persönliches Ansehen miteinander vereinte, setzte Brummell seine zwar nicht völlig neuartige, jedoch im historisch spezifischen Kontext des Niedergangs der traditionellen Ständegesellschaft offensichtlich besonders effektive, weil zumindest temporär als überlegen erscheinende Macht der ästhetischen Selbstinszenierung als Dandy entgegen. Indem die ästhetische Inszenierung hier ganz konkret der Machtausübung diente, kann der Dandy durchaus als Protagonist einer explizit ästhetischen Politik gelten.

Dabei erschien der Dandy zunächst einmal als problematischer Herd der Ansteckung und der Nachahmung hinsichtlich asozialen Verhaltens und exzentrischem Lebensstil, besonders plastisch in der zeitgenössisch omnipräsenten Beschreibung als Modegeck. Demgegenüber nimmt die Selbstbeschreibung des Dandys eine Umwertung vor, insofern er die eigene Unabhängigkeit, mithin die exzentrische Grundlage des Dandytums, als durch eine Ansteckung seitens des ,moralisierenden“ und sozialisierenden Massengeschmacks gefährdet erachtet. „Dandys stehen in einer geordneten und symmetrischen Gesellschaft für Willkür, sind aber in dem grauenhaften Puritanismus trotz aller Umsicht ansteckungsgefährdet." ${ }^{4}$ In sozialer und politischer Hinsicht war allerdings vor allem der berüchtigte Einfluss des Dandys auf die öffentliche Meinung problematisch. „Er war der Autokrat der Meinungsbildung.“5 Diesen öffentlichen Einfluss übte er dezidiert zynisch und verantwortungslos aus, und zwar nach der Maxime: „Bleibe in einer Gesellschaft, solange du noch keinen Eindruck gemacht hast; wenn du ihn gemacht hast, geh." ${ }^{6}$

Innerhalb der politischen Semantik der Demokratie fungiert der Dandy damit auch als Antipode des Revolutionärs: beide adressieren die Massen als ihr Publikum, der Dandy in ästhetizistischer und der Revolutionär in politischer Weise. Mithin verkörpert der Revolutionär die Dialektik, weil er das Leben in einer Utopie, die er als Aufhebung der Widersprüche und Gegensätze der bestehenden

4 Barbey d'Aureville: Über das Dandytum und über George Brummell, Berlin:

Matthes \& Seitz 2006, S.61.

5 Ebd.; S.5 1.

6 Ebd.; S.51. 
Wirklichkeit begreift, moralisch rechtfertigen will, während die pathetische Ästhetik der Differenz, der Distanz und der Vornehmheit eines zugleich zerstörenden und schaffenden Lebenskünstlers seinen Ausdruck in der Attitüde des aristokratischen Dandys findet. ${ }^{7}$ Der Dandy ist dabei anders als der Revolutionär, dessen Identität sich in der Entfaltung und utopischen Aufhebung eines absoluten Gegensatzes zu den herrschenden gesellschaftlichen Verhältnissen verwirklicht, keine anerkannte Identität, sondern eine sich immerzu ereignende und wiederholende Differenz zur modernen nivellierenden Gesellschaft ${ }^{8}$, aus der er keinen Weg hinausweist, von der er sich jedoch immer wieder auf vornehme und zynische Weise distanziert und vor deren Normen er immer wieder in die kalkulierten Leidenschaften des Ästhetischen flüchtet. Während sich der Revolutionär mittels der dialektischen Negation, der Aufhebung einer Entfremdung, verwirklicht, verherrlicht sich der Dandy in der differentiellen Bejahung, der Distanzierung. Der Dandy fragt nicht nach einem Grund, einem Wesen oder einer Identität, sondern nach der Art der Distinktion. Er rebelliert nicht auf politische, sondern auf ästhetische Weise. Nicht Moral oder gesellschaftliche Normen, mit denen er bricht und über die er sich hinwegsetzt, sondern Fragen des Stils prägen seine Revolte. ${ }^{9}$ Vornehmheit und Zynismus drücken eben keine kühle Indifferenz, sondern eine affektive und verzweifelte Beziehung ${ }^{10}$ zur modernen Gesellschaft, eine Attitüde der Verstellung ${ }^{11}$, aus, die sich weder auf ein Selbst noch auf die Gesellschaft reduzieren läßt. Im Unterschied zum Revolutionär ist, begründet und rechtfertigt sich der Dandy nicht; er erfindet, zerstört und schafft sich in der sich ereignenden und wiederholenden Provokation der bürgerlichen Gesellschaft ${ }^{12}$, in der verzweifelt zynischen, weil moralisch distanzierten Umwertung der (herrschenden) Normen. Während sich der Revolutionär als Spiegel der Gesellschaft identifiziert, um deren Widersprüche aufzuzeigen und auf ihre Aufhebung in einer neuen Gesellschaftsordnung hinzuarbeiten, exis-

7 Zur ästhetizistisch subversiven Figur des aristokratischen Dandys in der bürgerlichen Gesellschaft siehe Karl-Heinz Bohrer: Die Ästhetik des Schreckens. Die pessimistische Romantik und Ernst Jüngers Frühwerk, München: Hanser 1978, S.30-43. Vgl. auch Ellen Moers: The Dandy. From Brummel to Beerbohm, Lincoln: University of Nebraska Press 1978, S.288.

8 Vgl. Peter Heller: „Nietzsche über die Vornehmen und die Vornehmheit“, in: Peter Uwe Hohendahl und Paul Michael Lützeler (Hg.), Legitimationskrisen des deutschen Adels 1200-1900, Stuttgart: Metzler 1979, S.322.

9 Vgl. Richard Pine: The Dandy and the Herald. Manners, Mind and Morals from Brummel to Durrell, Basingstoke: MacMillan 1988, S.12f. u. S.24.

10 Zum verzweifelten Dandysmus Baudelaires vgl. Moers: Dandy, S.283.

11 Vgl. ebd., S.301.

12 Vgl. Pine: Dandy, S.12 u. $23 \mathrm{f}$. 
tiert der Dandy in der Form „leben und sterben vor einem Spiegel“ (Baudelaire). Repräsentiert der Revolutionär den negativen und reaktiven Nihilismus, so vollzieht der Dandy den Niedergang des Menschen als bejahender Nihilist, indem er aktiv selbstzerstörerisch wirkt und auf diese Weise das passive Dahinsterben des letzten Menschen in den schaffenden Akt der Zerstörung und Umwertung zu überführen versucht. „Le dandysme est le dernier éclat d'héroisme dans les décadences." 13

Der englische Dandy, der in der Figur George Beau Brummells in Frankreich durch Barbey d'Aureville gleichsam eingeführt und prominent gemacht wurde, zeichnete sich dadurch aus, dass er sich selbst und sein Leben - wie oben ausgeführt - zu einem regelrechten Kunstwerk stilisierte, während er hingegen darauf verzichtete, im konventionellen Sinne künstlerisch zu schaffen oder etwa zu schreiben. Dies änderte sich mit der französischen Adaption des Dandys, für die neben Barbey d'Aureville zunächst vor allem Charles Baudelaire stand. Während Barbey d'Aureville sein eigenes Dandytum gleichsam durch seine eher literarische als biographische Darstellung von George Brummell konstituierte, steht Baudelaires Dandytum ganz im Zeichen seiner ästhetischen Auseinandersetzung mit der modernen Gesellschaft. Die Figur des Dandys bildet daher auch einen zentralen Ausgangspunkt für Baudelaires viel rezipierte Diagnose von Modernität, die inhärent als liminal verfasst erscheint. Der Dandy figuriert dabei vor allem als metropolitaner Flaneur, also als genuin liminale Figur, die buchstäblich en passant die moderne Gesellschaft in der Metropole des 19. Jahrhunderts, Paris, beobachtet. ${ }^{14}$

Subjektivität ist für den passierenden Dandy also inhärent durch die Melancholie des selbsterzeugten und paradoxerweise stets gegenwärtig wahrgenommenen Verlusts geprägt. Ästhetisch veranschaulicht hat Baudelaire diese zugleich konstitutive und negative moderne Zeiterfahrung ebenfalls in seiner berühmten Beschreibung der subjektiven oder vielmehr überhaupt erst freilich ephemer subjektivierenden Wahrnehmung vorüber ziehender Wolken. Dieses Wahrnehmungsereignis manifestiert geradezu en passant die Unmöglichkeit, den Augenblick auch nur irgendwie festzuhalten oder gar zu erleben, ohne durch die Melancholie des darin inhärenten Verlusts affiziert und gleichsam infolge seiner je gegenwärtig bewussten Wahrnehmungen des Verschwindens als ephemeres Subjekt konstituiert zu werden. Hier wird der Dandy als Figur einer ausgesprochen liminalen Subjektivität konstituiert, und zwar

13 Charles Baudelaires: Le peintre de la vie moderne, in, ders.: CEuvres complètes, Paris: Gallimard 1961; S.1179.

14 Siehe hierzu auch Walter Benjamin: Das Passagenwerk, Frankfurt/Main: Suhrkamp. 
sowohl als zeitgenössisch gesellschaftlicher Typus wie auch als genuin literarische Figur. Das verbindende Element beider ist dabei eben die ästhetisierte Wahrnehmung der Welt, die sich insbesondere darin ausdrückt, dass der prekäre Schein als solcher und in seiner ephemeren Liminalität nicht nur gegenüber einem vermeintlichen Sein privilegiert, sondern im Sinne eines l'art pour l'art gegenüber einer realistischen oder naturalistischen Auffassung der Wirklichkeit dezidiert als einziger Modus der Konstitution von Welt rezipiert wird.

In Baudelaires Dandytum zeigt sich Liminalität zweifach, auf zwei verschiedenen Ebenen. Zum einen verkörpert der flanierende metropolitane Dandy par excellence die ephemere und liminale Modernität, wie Baudelaire sie hier metaphorisch beschreibt. Darüber hinaus ist bzw. wird der Dandy bei Baudelaire insofern liminal, als er ähnlich wie ein Schauspieler, allerdings bereits auf einer anderen Ebene, von einem Protagonisten der metropolitanen Wirklichkeit zu einer vor allem literarisch stilisierten Figur wird. In diesem ausgesprochen liminalen Sinn gehen Charakter und Form des Dandys bruchlos ineinander über. Denn der Lebensstil des Dandys erfuhr dergestalt eine literarische Wiederbeschreibung und Umschrift $\mathrm{zu}$ einem genuin literarischen Stil.

Darüber hinaus erlangt der Dandy bei Baudelaire ebenfalls eine ausgesprochen gesellschaftstheoretische bzw. sogar politische Dimension, indem er geradezu eklatant den prekären Übergang zwischen der aristokratischen zur bürgerlichen Gesellschaft verkörpert, wie in Baudelaires berühmter Definition des Dandys zum Ausdruck kommt:

Le dandysme apparaît surtout aux époques transitaires où la démocratie n'est pas encore toute-puissante, où l'aristocratie n'est que partiellement chancelante et avilie. Dans le trouble de ces époques quelques hommes declasses, dégoûtés, désoeuvrés, mais tous riches de forces natives, peuvent concevoir le projet de fonder une espéce nouvelle d'aristocratie, d'autant plus difficile à romper qu'elle sera base sur les facultés les plus précieuses, les plus indestructibles (...) Le dandysme est le dernier éclat d'héroisme dans les decadences. ${ }^{15}$

In gewisser Hinsicht epilogosiert der Dandy damit zugleich bereits die scheinbar zeitlose moderne bürgerliche Gesellschaft. 


\section{Décadence und Rhetorik des Endes}

Während der Dandy einen ausgesprochen ästhetischen Lebens-Stil pflegte, zeichnet Friedrich Nietzsche ein ästhetisch geprägter DenkStil aus. Paradigmatisch wird diese stilistische Affinität des Dandys zur Philosophie Nietzsches paradoxerweise in der differenten Haltung zum Problem der Dekadenz. Im sogenannten Fin de Siècle verkörperte der Dandy wohl par excellence dasjenige Phänomen, das ausgehend vom zeitgenössisch kulturkritischen Diskurs um die Degeneration der Gesellschaft als décadence bezeichnet wurde. Insbesondere in der Literatur avancierte diese Kategorie in der Figur des Dandys zu einem Leitmotiv, für das Joris Karl Huysmans' Des Esseintes und vor allem Oscar Wildes Dorian Gray die Protagonisten darstellten. Diese sogenannte décadence-Literatur resignifizierte den zeitgenössisch virulenten Diskurs der Degeneration, wie er z.B. in Max Nordaus Werk „Entartung“ unter expliziter Einbeziehung der zeitgenössischen Kunst und Literatur artikuliert wurde, ästhetizistisch. Dabei fungierte der Dandy, verstärkt in die Nähe zum Verbrecher gerückt, geradezu als emblematische Figur dieser ästhetizistischen Resignifikation des weitgehend kulturkritischen Diskurses der Degeneration in der Literatur.

Auf diese Weise geriet der literarisch stilisierte Dandy geradezu zur Stilikone der décadence des Fin de Siècle. Mehr noch, der Dandy, wie er sich im 19. Jahrhundert zunächst in England herausgebildet hatte und zur Jahrhundertwende insbesondere in Frankreich zu einer literarisch prominenten Figur zeitgenössischer Selbstbeschreibungen wurde, stilisierte in einer selbstreferentiellen Operation den Stil (selbst) zu einer polyvalenten Form, ja zu einem regelrechten Medium, ästhetisch-ethischer Subjektivierungen. Im Medium des Stils und in der komplementären Form des zumeist sinnlichen Ereignisses, das der Dandy gleichermaßen sucht und evoziert, konstituiert er seine durch und durch ästhetische Existenz, denn „(...) auf Intensität des seltenen Erlebnisses aus, setzt dieser ,Stil den Autonomieanspruch des Ästhetischen gegenüber dem ,Leben“ absolut."16 Damit verbinden sich Aggressivität und ein subversiver, transgressiver Gestus des Stils, die das Leben keineswegs ästhetisch aufheben, sondern es ästhetizistisch überschreiten.

Ganz in diesem dandystischen Sinne formulierte Oscar Wilde zeitgenössisch: „to think is to act"17, wobei Denken und Handeln hier gleichermaßen mit dem Index des ethisch-ästhetisch Transgressiven oder gar Subversiven belegt werden. Dies rückt wiederum den Dandy mit seiner Affinität der bewussten Überschrei-

16 Bohrer: Ästhetik, S.26.

17 Vgl. ebd., S.31. 
tung gesellschaftlicher Normen ausdrücklich in die Nähe des Verbrechers, wie literarisch besonders ausgeprägt bei Wilde und Huysmans im Fin de Siècle ${ }^{18}$ und damit auch im Kontext des zeitgenössischen Diskurses der Degeneration. Hierfür steht wiederum paradigmatisch Oscar Wildes Figur des Dorian Gray, der sein jugendliches Aussehen, seine Schönheit, bewahrt, indem er durch den Ausspruch, das von seinem Künstlerfreund angefertigte Bildnis seiner selbst solle an seiner statt altern, gleichsam seine Seele in sein Bildnis transponiert, das fortan entsprechend durch die Spuren seiner unmoralischen Ausschweifungen hässlich entstellt wird, während er selbst in seinem Aussehen seine jugendliche Unschuld bewahren kann. Dabei handelt es sich nicht nur um die für die künstlerische Moderne des Fin de Siècle so programmatische Konfrontation zwischen Leben und Kunst, sondern darüber hinaus um eine immanente Verbindung von Literatur und Kunst hinsichtlich der ästhetischen Dekonstruktion des modernen Subjekts, also um einen dezidiert ästhetischen Diskurs der Moderne.

Der ausdrücklich gegen ethisch begründete gesellschaftliche Normen gerichtete sogenannte Ästhetizismus konstituiert dabei diese im Fin de Siècle besonders ausgeprägte Affinität und Liminalität zwischen Dandy und Verbrecher, d.h. „...dass ästhetische Konzentration an sich schon die Tendenz zur moralischen Empfindungslosigkeit, ja zur Grausamkeit in sich trägt."19 Und weiter:

(...) wir erkennen einerseits zwar, dass die weitere Geschichte des europäischen Ästhetizismus und des in ihm aufkommenden Motivs des ,Schreckens" in solch einem Satz seinen Anfang nahm; wir erkennen gleichzeitig aber, dass Ruskins Aufforderung an die künstlerische Konzentration nur einen Gedanken fortsetzt, der von der Tradition der ästhetischen Diskussion seit Ausgang des 18. Jahrhunderts legitimiert ist: danach unterscheidet sich der Künstler notwendigerweise vom Bürger dadurch, dass er sinnliche Phänomene nach Maßgabe ihres künstlerischen Effekts betrachtet. Eine solche ästhetische Konzentration, deren Wert durch ethische Interessen herabgemindert werden könnte, läuft Gefahr, das Inhumane, das Böse zu beschwören in dem Augenblick, wo durch besondere historische Umstände der Gegenstand des künstlerischen Interesses ein inhumaner Gegenstand ist. ${ }^{20}$

Was gerade Nietzsche schließlich mit dem kulturellen, vor allem literarischen, Fin de Siècle verbindet, ist eine häufig mythisch anmutende Rhetorik des Endes. Das Ende, der Untergang, der modernen Gesellschaft und des Menschen ist bei ihm die Bedingung und Vor-

18 Vgl. ebd., S.32.

19 Vgl. ebd., S.57.

20 Vgl. ebd., S.57f. 
aussetzung für den „Aufstieg zum Übermenschen“..21 Dieser wird allerdings nicht identifiziert, sondern bejaht sich allein in seiner Differenz und Distanz zum Menschen. Mithin schafft Nietzsche keine erkennbare Vision, die über die Zerstörung des Bestehenden hinausgeht. Hierin äußert sich eine radikale Metaphysikkritik, die auch der literarischen Moderne des Fin de Siècle in Form apokalyptischer Bilder $^{22}$ eigen war. Werden bei Nietzsche Dekadenz und Zerstörung als notwendige, den Willen zur Macht von einer nihilistischen Moral befreienden, Voraussetzungen zur Entfesselung einer dionysischen Schöpferkraft des Lebens diesseits metaphysischer Vorstellungen aufgefasst, so regt die Rhetorik des Weltuntergangs innerhalb der modernen „Dekadenzliteratur“ experimentelles und nervöses künstlerisches Schaffen an. ${ }^{23}$ Insgesamt artikuliert sich die Rhetorik des Endes im Fin de Siècle weniger resignativ als verzweifelt expressiv. Sie mündet auf dieser Ebene in vielfältige Formen einer Flucht, die allein in der verzweifelten Suche nach ästhetischer Erfahrung und Originalität des Stils eine gemeinsame Richtung anzeigen.

Allerdings endet diese Flucht vor der bürgerlichen Massengesellschaft, ihrer herrschenden „Kultur der Anerkennung“, die sich in der nihilistischen Rechtfertigung des Lebens anhand absoluter höherer Werte und etablierter Normen vollzieht, und ihrer nivellierenden Atmosphäre bei Nietzsche wie bei den Vertretern der literarischen Moderne in der „Attitüde des Einsamen, der den letzten Rest verteidigt, der ,von dem Anspruch auf Autonomie des bürgerlichen Subjekts noch übrig blieb“."24 In den von der modernen Zivilisation abgewandten Einsiedler (Zarathustra) projiziert Nietzsche den keineswegs bruchlosen, sondern in der ewigen Wiederkehr sich zugleich wiederholenden und verwandelnden, Übergang zum Übermenschen. Auch Nietzsches Wahnsinn erscheint wie die ästhetizistische Innerlichkeit moderner Literaten als ein letzter Fluchtweg, der aus Verzweiflung über die morbide Lebensfeindlichkeit der bürgerlichen Gesellschaft und deren Normen das Pathos der Einsamkeit dem differenzlosen Aufgehen im Ganzen, das Distinktion nur noch als repräsentativen Status (des Seins) und nicht mehr in ihrem zufälligen Werden zuläßt, vorzieht. Denn der Wille zur Macht kann sich wie die Vornehmheit nur als singuläres zufälliges Werden, das sich in der Differenz bejaht, äußern, nicht als allgemeines rationalisiertes Streben nach Erfolg und Macht, letztlich also nach

21 Vgl. Nietzsche: Also sprach Zarathustra. Ein Buch für Alle und Keinen, 1883 , S.10ff.

22 Vgl. Wolfgang Lange: „Im Zeichen der Dekadenz. Hofmannsthal und die Wiener Moderne“, in :Rolf Grimminger (Hg.), Literarische Moderne. Europäische Literatur im 19. und 20. Jahrhundert, Reinbek: Rowohlt 1995, S.204f.

23 Vgl. ebd., S.202ff.

24 Vgl. Bohrer: Ästhetik, S.43. 
Etablierung, Anerkennung und Repräsentation ${ }^{25}$, das die bürgerliche Gesellschaft und den modernen Staat charakterisiert.

Anders als die literarische Moderne, deren Flucht in die rein ästhetische Erfahrung einen „kalkulierten Wahnsinn“26 zum Ausdruck bringt, der grundsätzlich reversibel ist und zumeist lediglich eine Phase künstlerischen Schaffens prägt, ist Nietzsches stilisierte Schizophrenie die äußerste Konsequenz aus der verzweifelten Affektion seines zugleich egozentrischen und fragmentierenden Denkens und Schaffens, das insgesamt auf eine ästhetische Umwertung der Werte abzielte, und zwar vor allem in der literarischen Form des Aphorismus, die in den späteren Schriften Nietzsches gleichsam den diskursiven Stil par excellence des radikal umwertenden Wahns bildete. Denn diese Schizophrenie erscheint geradezu als ästhetisch verfahrende Dekonstruktion bürgerlicher Identität.

Kein Nietzsche-Ich, Professor der Philologie, verliert urplötzlich seinen Verstand und identifiziert sich mit fremden Personen, vielmehr durchläuft das nietzschesche Subjekt eine Serie von Zuständen und identifiziert die Namen der Geschichte mit diesen Zuständen: jeder Name der Geschichte bin ich(...) Um den Kreis herum, aus dessen Zentrum das Ich desertiert ist, breitet sich das Subjekt aus. Im Zentrum steht die Wunschmaschine, die zölibatäre Maschine der ewigen Wiederkunft. Das nietzschesche Subjekt, Residualobjekt der Maschine, zieht euphorischen Gewinn (Voluptas) aus allem, was diese in Gang setzt, und von dem der Leser angenommen hatte, dass es allein das in Fragmenten vorliegende Werk Nietzsches gewesen sei. ,Nietzsche glaubt von nun an nicht die Realisierung eines Systems, sondern die Anwendung eines Programms zu betreiben.' Nicht mit Personen sich identifizieren, wohl aber die Namen der Geschichte mit Identitätszonen auf dem organlosen Körper identifizieren(... ${ }^{27}$

\section{Nietzsches ästhetische „Umwertung der Werte“}

In seiner „Genealogie der Moral“28 rekonstruiert Nietzsche die Geschichte eines Nihilismus im religiösen und philosophischen Denken. Nihilismus steht dabei für die Entwertung und Verneinung des Lebens vom Standpunkt absoluter Werte aus. Diese Entwertung und Verneinung erfolgt dadurch, dass das Leben an sich moralisch unter Anklage gestellt wird und schließlich nur anhand höherer

25 Vgl. zur Nichtigkeit repräsentierter Macht, Deleuze: Nietzsche, S.88ff.

26 Siehe Wolfgang Lange: Der kalkulierte Wahnsinn. Innenansichten ästhetischer Moderne, Frankfurt/Main: Fischer 1992.

27 Gilles Deleuze/Félix Guattari: Anti-Ödipus. Kapitalismus und Schizophrenie, Bd.I, Frankfurt/Main: Suhrkamp 1974, S.29f.

28 Siehe Zur Genealogie der Moral. Eine Streitschrift, Leipzig 1887, Kritische Studienausgabe, Bd.5, hrsg. v. Giorgio Colli u. Mazzino Montinari, Berlin: de Gruyter 1993, S.245ff. 
Werte gerechtfertigt werden kann. Leben wird durch die nihilistische Tradition als ungeordnetes, chaotisches und böses Tun der Menschen interpretiert, dem erst durch verbindliche Moral- und Wertvorstellungen ein Sinn verliehen werden kann. Das Leben wird stets zunächst schuldig gesprochen, um es dann von dieser Schuld zu erlösen.

Ausgangspunkt der Genealogie ist die Frage nach Vornehmheit oder Niedrigkeit der Herkunft der Werte und ihrer jeweiligen Kräfte. Allerdings handelt es sich bei dieser Dichotomie um keine Dialektik des Negativen; die niedrige "Sklavenmoral“ ist gerade dadurch charakterisiert, sich sklavisch den herrschenden Werten unterzuordnen, sie absolut zu setzen und sie gleichzeitig vermittels einer negativen Dialektik zu kritisieren, indem eine Entfremdung von den absoluten Werten moniert wird, deren Grund das Böse als Negation des Guten abgibt. Dem Guten als absolutem Wahren wird dabei das Böse als Entfremdung dieses Guten in Form des Lebens gegenübergestellt. Das Leben wird mithin zur Manifestation der Entfremdung durch das Böse schlechthin erklärt, indem es an den metaphysisch vorgestellten absoluten Werten des Guten gemessen wird. Um gegenüber dem Leben wirksam zu werden, muß sich die moralische Bewertung dieser niedrigen Sklavenmoral jedoch negativ und reaktiv äußern. Auch wenn sie dem Leben in Form von absoluten Werten vorgestellt wird, kann die Metaphysik sich immer nur retrospektiv dem Bösen entgegenstellen. „Du bist böse, also bin ich gut.“ Das Gute wird zur Negation, Aufhebung und Reflexion des Bösen. Das Gute muß dabei vorausgesetzt werden, um sich reaktiv als Negation der Entfremdung durch das Böse zu begründen. Die absoluten Werte, die das Leben begründen, rechtfertigen und ihm einen Sinn geben, müssen es zunächst entwerten und negieren. Hierin sieht Nietzsche den verneinenden Willen zur Macht, der dadurch charakterisiert ist, dass er die aktiven Kräfte von dem zu trennen anstrebt, was diese können.

Dagegen sind Vornehmheit und Herrenmoral bejahende Existenzweisen, die sich nicht absoluten und etablierten Werten unterwerfen, sondern stets Werte schaffen. Das Leben wird hier nicht reaktiv anhand absoluter Werte beurteilt, sondern drückt sich aktiv in der Schaffung neuer Werte, in der Umwertung, aus. Dabei erfolgt keine negative Gegenüberstellung von Gut und Böse, sondern Vornehmheit geht aus der Differenz, die sich in den Wert- und Kräftekonstellationen ausdrückt, hervor. Bejahung des Lebens bedeutet dabei nicht, es als absoluten Maßstab eines Seins zu betrachten, sondern es sich als zufälliges (differentielles) Werden im Sinne der ewigen Wiederkehr ständig neu schaffen, ereignen und wiederholen zu lassen. Bejahung heißt in diesem Zusammenhang also Schaffung von Werten aus dem Leben heraus, so dass es keines Schöp- 
fers, keiner Metaphysik und keiner absoluten Moral bedarf. An die Stelle der absoluten moralischen Kategorien gut und böse treten die Differenzen der Herkunft von Werten wie vornehm-niedrig, gutschlecht oder aktiv-reaktiv. Dies sind keine absoluten Kategorien, sondern relative, differentielle Werte, die immer in Affinität zu den entsprechenden Kräften und dem entsprechenden Willen zur Macht gesehen werden. Ein Wert ist nicht absolut gut, sondern wird als schaffender, bejahender, befehlender und aktiver Wert gut, während er als verneinender, gehorchender und reaktiver Wert schlecht wird. Ein konventionell absoluter Wert wie z.B. der Fleiß kann somit als schaffender, befehlender und bejahender Flei $\beta$ gut, als gehorchender und reaktiver Fleiß jedoch schlecht werden. Alles hängt davon ab, welcher Willen zur Macht und welche Kräfte sich in den Werten äußern: bejahende oder verneinende. Während der verneinende und reaktive Wille zur Macht die aktiven Kräfte von dem trennen will, was diese können, um selbst zu sein, will der bejahende, schaffende und aktive Wille zur Macht sich aus dem Leben heraus äußern. Der verneinende Wille zur Macht will das Leben beherrschen, indem er es verneint; der bejahende Wille zur Macht will sich ständig aus dem Leben heraus verherrlichen, indem er es bejaht. Das Prinzip des ersten ist die Negation des Lebens, während der zweite durch die Bejahung der Differenz das Leben an sich bejaht. „Ich bin gut, also bist du schlecht.“ In dieser Aussage drückt sich die Differenzen bejahende Vornehmheit der Herrenmoral aus.

Letztlich tritt in Nietzsches Genealogie der Moral an die Stelle absoluter Werturteile oder Werte eine Rekonstruktion der konstitutiven Kräftekonstellationen, der differentiellen Relationen der (herrschenden) Werte, den in ihnen wirksamen Kräften und des korrespondierenden Willen zur Macht. Die Bewertung der Werte erfolgt hier nicht anhand absoluter Kategorien, sondern anhand der Wertund Kräfterelationen, die bestimmte Werte in der Geschichte etablieren. Von der Ideologiekritik unterscheidet sich diese Genealogie dadurch, dass sie keinen absoluten Wert im Sinne eines privilegierten Standpunktes mehr zulässt. Die Bewertung der Werte geht aus ihrer Herkunftsgeschichte in Relation zu anderen Werten und Kräften hervor und in der Differenz zwischen vornehm und niedrig auf. Nietzsche fasst Werte nicht als Entitäten, sondern als Kräftekonstellationen auf. Der jeweilige Wille zur Macht ist dabei keine absolute Kategorie, kein einheitlicher Zustand, sondern eine Differenz zwischen einer reaktiven (nicht passiven) Rückführung des Lebens auf einen letzten Grund, wodurch der verneinende Wille zur Macht in der nihilistischen Tradition charakterisiert ist, und einer aktiven Bejahung des Lebens als zufälliges, unschuldiges und nicht $\mathrm{zu}$ rechtfertigendes Sein, das sich in der ewigen Wiederkehr manifestiert. 
Die dialektische Philosophie wird auf diese Weise als Herrschaft der reaktiven Kräfte einer niedrigen Sklavenmoral, die die aktiven Kräfte von dem trennt, was diese können, erklärt und entlarvt. Die dialektische Metaphysik des absoluten Guten, das das Böse negiert, aufhebt und sich in dieser Bewegung vervollkommnet, wird bei Nietzsche durch eine Wissenschaft von Differenzen und Relationen, die das Absolute untergraben, zerstört. Die absoluten moralischen Kategorien von Gut und Böse sollen anhand der relativen, an den affektiven Instinkten gemessenen, Bedingungen ihrer vornehmen oder niedrigen Herkunft bewertet werden. Hierbei ist das Leben nicht mehr das bloße Objekt einer ursprünglichen Schöpfung oder metaphysischen Moral, sondern als schaffendes Sein das einzige, sich ereignende und sich wiederholende Subjekt und Schöpfer aller Werte.

Nietzsches Denken ist eine einzige Kampfansage an die Philosophie des negativen Absoluten vor allem der Hegelschen Dialektik. Nietzsche greift das klassische dialektische Verhältnis zwischen Herr und Sklave auf, um zu zeigen, dass es die erfolgreiche Projektion des siegreichen Sklaven darstellt. Demnach beruht dieses Verhältnis auf dem unvermeidlichen Sieg der Sklaven im „Kampf ums Dasein“, da diese auch als Herren immer Sklaven der Anerkennung in diesem dialektischen Verhältnis bleiben. Anerkennung resultiert aus der negativen Rückführung der Differenz auf einen Grund. Dialektik ist Ausdruck der reaktiven Kräfte, eines verneinenden Willens zur Macht, der eine Differenz der Herkunft auf einen absoluten Gegensatz zurückführt, so dass schließlich sowohl Sklave als auch Herr Sklaven der negativen Anerkennung bzw. der wechselseitigen Negation sind. Der Herr negiert den Sklaven, um Herr zu sein, während der Sklave den Herren negiert, um selbst Herr zu werden. Indem sich beide allerdings des negativen Gegensatzes versichern, versuchen sie auf nihilistische Weise ihre Existenz zu rechtfertigen. Die existentielle Differenz der Herkunft und das Leben als Zufall werden verneint, um auf der Negation die sklavische Bejahung in Form der Anerkennung innerhalb eines absoluten Gegensatzes zu begründen. Die dialektische Auseinandersetzung, der Kampf zwischen Herr und Sklave, ist mithin ein reaktives und nihilistisches Verhältnis, dem ein verneinender Wille zur Macht innewohnt und das stets den Sieg der Sklaven mit sich bringt, da es sich in der Negation rechtfertigt.

Einer derartigen dialektischen Sklavenmoral stellt Nietzsche eine Herrenmoral gegenüber, die sich in einem aristokratischen $\mathrm{Pa}$ thos der Distanz und der Vornehmheit (der Herkunft) ihren Ausdruck verschafft. ${ }^{29}$ Distanz und Vornehmheit gehen dabei nicht aus

29 Vgl. Nietzsche: Jenseits von Gut und Böse. Vorspiel einer Philosophie der Zukunft, Leipzig 1885, Kritische Studienausgabe, Bd. 5, hrsg. v. Giorgio 
der Negation oder sklavischen Anerkennung, sondern aus einer Bejahung der Differenz, der Selbstverherrlichung, hervor. Das Leben und die Differenz werden nicht auf nihilistische und reaktive Weise gerechtfertigt oder auf einen Grund zurückgeführt, sondern in ihrem zufälligen Werden bejaht. Während das dialektische Verhältnis zwischen Herr und Sklave in der wechselseitigen Negation gründet, entziehen sich Distanz und Vornehmheit pathetisch, d.h. zugleich irrational und vital, jeder Notwendigkeit eines letzten Grundes. Ein derartig affirmatives Denken der Differenz rechtfertigt sich nicht in der negativen Entfaltung eines absoluten Gegensatzes innerhalb eines identischen und anerkannten Seins, sondern strebt nach einem „Aufstieg ins Grundlose“ eines nicht identifizierbaren und nicht (moralisch) zu rechtfertigenden Werdens. Während die Dialektik eine an absoluten Werten ausgerichtete moralische Beurteilung und Rechtfertigung des Lebens vornimmt, findet bei Nietzsche eine ästhetische und schaffende Anschauung des Lebens in der Distanz und Verstellung ihren Ausdruck. Ästhetik ist dabei keine moralisch gebundene Kategorie wie im bürgerlichen Humanismus, sondern die, über die Zerstörung des Bestehenden hinaus, schaffende Tätigkeit eines Künstlers, der nicht nach herrschender Anerkennung seines Werkes, sondern nach dionysischer Ekstase im pathetischen, weil kankhaft-vitalen Schaffensproze $\beta$ selbst strebt.

In diesem Sinne führt Nietzsches Denken der Differenz zu einem ästhetischen, vitalistischen und schließlich aristokratischen Dandysmus, der die bürgerliche Werte- und Gesellschaftsordnung mitsamt ihren Normen provoziert, ohne sie auf revolutionäre Weise zu überwinden oder aufzuheben.

\section{Der Wille zum Stil}

Die Attraktivität dieses Denkens bestand weniger in seinen inhaltlichen Aussagen als in einem machtvollen, aggressiven, manchmal zynischen, häufig egozentrischen, im wahrsten Sinne des Wortes radikalen, weil scheinbar mit allem Bestehenden tabula rasa machenden Stil. Mit diesem Stil wurde Nietzsche zum Modephilosophen des Fin de Siècle. ${ }^{30}$ Seinem Denken schien es weniger um politische Inhalte als um einen ästhetisch im dionysischen Sinne verfahrenden Umgang mit diesen zu gehen. Nietzsche konnte für die verschiedensten politischen Richtungen instrumentalisiert werden, da seine Wahrnehmung der Wirklichkeit nicht moralisch oder gar

Colli u. Mazzino Montinari, Berlin: de Gruyter 1993, S.9ff.; S.215 u. Peter Heller: Nietzsche, S.322.

30 Vgl. Steven E. Aschheim: The Nietzsche Legacy in Germany 1890-1990, Berkeley: University of California Press, S. 24. 
politisch, sondern ästhetisch ausgerichtet war. „(...)denn nur als ästhetisches Phänomen ist das Dasein und die Welt ewig gerechtfertigt". 31

Dabei bricht Nietzsches Ästhetik allerdings mit den ästhetischen Vorstellungen der humanistischen Tradition, indem sie an Baudelaires Ästhetik des Hässlichen anknüpft und diese in eine gegen die humanistische Moral gerichtete Ästhetik des Bösen überführt. Sowohl die Ästhetik des Hässlichen als auch die Ästhetik des Bösen markieren eine Flucht vor den, am Absoluten Hegelscher Prägung ausgerichteten, bürgerlichen Kategorien des Schönen und des Guten, indem sie die Dialektik von Schön und Hässlich bzw. von Gut und Böse untergraben und das Hässliche bzw. das Böse nicht in einer Negation, sondern in der bejahten untergründigen Differenz (selbst) verorten. ${ }^{32}$ Diese Flucht eröffnet mehrere, sich wechselseitig brechende, Fluchtlinien in Richtung Ästhetizismus und Avantgarden.

In der dezidiert „antipolitischen“ zynischen Attitude Nietzsches steckt also gerade seine politische Brisanz und die ideologische Sprengkraft, die sich in der Rezeption manifestiert. Denn der Extremismus Nietzsches äußert sich nicht etwa in politischen Überzeugungen, sondern vor allem in der ästhetischen Distinktion von vornehm und niedrig sowie in der Intensität der ästhetischsinnlichen Affirmation weltlicher Erfahrung oder einfacher: des Lebens, wie sie die Begriffe amor fati und ewige Wiederkehr zum Ausdruck bringen. Im Verhältnis zum neuzeitlich abgesteckten Territorium des Politischen verhält sich dieses Denken nomadisch, da es sich selbst nicht ideologisch ummauert oder politisch manifestiert. Nietzsche schuf keine einheitliche politische Ideologie, sondern eine stilistische „Kunst“ der radikalen, weil nicht mehr dialektisch negativen, Kritik. Dabei wirkt er weniger polemisch, auf eine offene Auseinandersetzung abzielend, als zynisch und agonistisch, da er den Niedergang, das „Zugrundegehen“, des Menschen herbeisehnt, um den „Übermenschen“ zu ermöglichen. Der Übermensch verkörpert allerdings keineswegs eine inhaltliche (soziale) Utopie, sondern den formalen Bruch mit allen, zwangsläufig dialektischen, Utopien und die Befreiung des Lebens von allen reaktiven und verneinenden Kräften wie sie der Mensch mit seiner Moral, seiner Kultur und seiner Geschichte verkörpert. Während der Mensch, durchdrungen von der reaktiven Herrschaft höherer Werte, metaphysischer Sinnhaftigkeit und absoluter Moral, das Leben an sich verneint, um es

31 Vgl. Friedrich Nietzsche: Die Geburt der Tragödie aus dem Geiste der Musik, Leipzig 1872, Kritische Studienausgabe, Bd.1, hrsg. v. Giorgio Colli u. Mazzino Montinari, Berlin: de Gruyter 1993, S.43.

32 Siehe hierzu auch Gilles Deleuze: Differenz und Wiederholung, München: Fink 1992, S.50f. 
nachträglich inhaltlich zu rechtfertigen, bejaht der Übermensch das Leben an sich als schaffende Form einer genealogisch und stilistisch vornehmen Gattungstätigkeit.

Letztlich war es diese Fokussierung auf Stil und Form, die Nietzsches Denken im Fin de Siècle, das durch ein „europäisches Wert-Vakuum“33, eine Krise der Inhalte bürgerlicher Moral und Normen, charakterisiert werden kann, so attraktiv machte. Auch und gerade in Kunst und Literatur der Jahrhundertwende läßt sich als Antwort hierauf eine „Ästhetisierung aller Lebensbereiche“34, eine Tendenz der zunehmenden Betonung von Stil und Form gegenüber Inhalten ausmachen, die in Baudelaire ihren Vorläufer hatte. ${ }^{35}$ Das Fin de Siècle schien insgesamt ein Ende der inhaltlich verbindlichen (kategorischen) Imperative aus der Aufklärung zu markieren. Während in der Kunst und Literatur die zunehmende Fragmentierung der Stile bei gleichzeitigem Bedeutungsverlust der Inhalte ein Indiz hierfür darstellte, relativierte Nietzsches Denken radikal die fundamentalen Prämissen der Aufklärung. Mithin treten im Fin de Siècle in den verschiedensten Bereichen Differenzen (des Stils) an die Stelle der vormals verbindlichen Dialektik von Inhalt und Form. ${ }^{36}$ Nietzsche geht dabei noch weiter, insofern er nicht einfach einen neuen Stil etabliert, sondern die Distinktion der Stile in Form der Bejahung von Differenz und anhand vornehmer oder niedriger Kräfterelationen schafft. Auch im Bereich der Stilistik zeichnet Nietzsche Fluchtlinien und führt Unterbrechungen ein, die sich jeder Herrschaft eines einheitlichen Stils entziehen. Dabei ist der Stil für ihn das über das „Gefährliche in Dingen und Worten tapfer Hinwegspringende“. 37

Diese Fluchtbewegung stie $\beta$ in einer, durch eine zunehmende Dichte herrschender Konventionen durchdrungenen, Massengesellschaft, aus der das Verlangen nach Distinktion im Fin de Siècle immer wieder singulär hervortrat, auf fruchtbaren Boden. Dies äußerte sich vor allem kulturell, besonders in Kunst und Literatur, in einer distinktiven Flucht ${ }^{38}$ vor den Manifestationen der nivellierenden, morbid und lebensfeindlich erscheinenden Massengesellschaft. Im Vordergrund stand dabei der Bruch mit der utilitaristischen Le-

33 Vgl. Lange: Wiener Moderne, S.204.

34 Ebd.; S.204.

35 Vgl. Peter Bürger: Theorie der Avantgarde, Frankfurt/Main: Suhrkamp 1974, S.25f. und zur Aggressivität des ästhetizistischen hermetischen „Stilpathos“ vgl. Bohrer: Ästhetik, S.26f.

36 Vgl. Bürger: Avantgarde, S.25.

37 Vgl. Bohrer: Ästhetik, S.63.

$38 \mathrm{Zu}$ ästhetizistischem Eskapismus und Fluchtmotiv in der Moderne siehe ebd., S.43f. 
benspraxis ${ }^{39}$, der sich sowohl in einer ästhetizistischen Innerlichkeit als auch in expressiven avantgardistischen Bewegungen äußerte. Auf jeden Fall markieren Baudelaires Ästhetik des Hässlichen und Nietzsches Ästhetik des Bösen die Unterbrechungen und Fluchtbewegungen über den Horizont des bürgerlichen Humanismus hinaus, die sich in den ereignishaften, aggressiven und gefährlichen Manifestationen einer „Ästhetik des Schreckens“40 gegenüber den tradierten Konventionen von Erhabenheit und humanistischer Bildung und Erbauung artikulierten.

Der oben skizzierten bürgerlichen Variante des Nihilismus, der Orientierung an absoluten Werten (Hegel) und der zunehmend unbeantwortbar erscheinenden Sinnkrise dieser Werte im Fin de Siècle (Schopenhauer) setzt Nietzsche eine vitalistische, bejahende und schaffende Lebensauffassung gegenüber, die in offene Distanz zur bürgerlichen Mediokrität tritt und mit einem radikalen aristokratischen Gestus versehen ist. Aristokratisch deshalb, weil er der bürgerlichen Gesellschaft eine sklavische Unterwürfigkeit und Schwäche vorwirft, die sich in Idealen wie christlicher Nächstenliebe, bürgerlicher Humanität und sozialistischer Brüderlichkeit äußert. Dagegen richtet er eine herrische Arroganz und zynische Distanzierung in aristokratischer Manier. Nietzsches Aristokratie ist dabei nicht einfach nur politisch reaktionär oder sozial elitär, sondern zeichnet sich durch eine Kultur der Distinktion aus, die ihren Ausdruck in einem ästhetisch-singulären, d.h. antirationalem und antidemokratischem, Pathos der Distan $z^{41}$ einer subjektivistischen Vornehmheit findet. Aristokratie bezeichnet hier also weder eine politische Einstellung noch eine soziale Klasse, sondern ein distinktives, in Differenz zur bürgerlichen Massengesellschaft sich artikulierendes, Pathos der vitalen Singularität des Seins diesseits der vorgestellten Identität des rationalen (bürgerlichen) Individuums.

Seine aristokratische Vitalität bricht dabei radikal mit dem, aus der Aufklärung hervorgegangenen, Horizont bürgerlicher Werte, Normen und Konventionen, ohne einfach nur romantisch zum Mythos zurückzukehren. Im Unterschied zur Romantik beantwortet Nietzsche das Symptom der „Krise“ des Individuums und seiner Identität in der modernen bürgerlichen Gesellschaft infolge der vielfach als Entfremdung wahrgenommenen „Rationalisierung“ nicht mit dem Postulat einer Einheit von Körper und Geist, sondern indem er, bestrebt die vitalen menschlichen Instinkte in Form eines bejahenden Willen zur Macht freizulegen, den rationalen Geist als Äußerung einer reaktiven Kraft, eines verneinenden Willen zur Macht, der sich durch die nihilistische Entwertung, die Trennung

39 Vgl. Bürger: Avantgarde, S.67.

40 Vgl. Bohrer: Ästhetik, bes. S.22f.

41 Vgl. Nietzsche: Jenseits von Gut und Böse, S.215. 
der aktiven physischen Kräfte von dem, was diese können, zu einer metaphysischen Herrschaft aufschwingt, auf die physischen Kräfte und den Körper zurückführt. Der Körper stellt dabei jedoch keine (physische) Identität dar, sondern wird als vitale Macht, als relationales Kräfteverhältnis, das über Vornehmheit oder Niedrigkeit der Herkunft aussagt. Die Figur des Dandys, die sich durch eine (oberflächliche) Ästhetisierung des Körpers, der Form, bei gleichzeitiger Herabsetzung geistiger Inhalte auszeichnet ${ }^{42}$, bildet im Fin de Siècle, an das Leben und Werk Baudelaires anknüpfend, einen Fluchtpunkt dieser antirationalistischen Philosophie.

Vornehmheit stellt Nietzsches Antwort auf die nihilistische Dekadenz und Degeneration der bürgerlichen „Sklavengesellschaft“ dar. In dieser Vornehmheit drückt sich jedoch nicht ausschließlich eine egozentrische Distinktion, sondern auch eine radikale antipolitische Attitüde aus. Nietzsches Distanzierung von der Gesellschaft erfolgt nicht in revolutionärer, auf eine effektive Umwälzung der gesellschaftlichen Ordnungs- und Wertesysteme ausgerichtete, Weise; Nietzsche kann nicht zu einem Revolutionär, auch keinem reaktionären, gemacht werden. Stattdessen distanziert er sich von jeder, wenn auch nur utopischen, Vision von Identität, indem er sich vom politisch-repräsentativen, demokratischen und mehrheitlichen Charakter der modernen Utopien abwendet. Gerade in diesen macht er ja die dialektisch daherkommende Herrschaft von Sklavenmoral und absoluten Werten aus, die das Leben verneinen und entwerten. Hierin zeigt sich eine egozentrische Abgehobenheit, die aus der Sicht einer, den verbindlichen Normen des Christentums oder der (politischen) Aufklärung verschriebenen, Massengesellschaft von Bürgertum und Arbeiterschaft als zynisch wirken muß, da sie statt einer Überwindung der auch hier vielfach wahrgenommenen Krise der Gesellschaft deren alternativlosen und notwendigen Niedergang herbeisehnt.

Andererseits wird dieses Denken im Fin de Siècle für nahezu alle Varianten selbsternannter Avantgarden zu einem Ort der Sehnsucht nach Distinktion und Vornehmheit im Verhältnis zur anonym, morbid und lebensfeindlich erscheinenden Massengesellschaft. Gerade im Bürgertum, dessen ökonomischer Erfolg mit einer Form niedriger Mediokrität in kultureller Hinsicht einherzugehen scheint, setzen Nietzsches vitalistische Auffassungen von Vornehmheit und Distinktion kompensatorische Marken vor allem für jugendlich Gesinnte, denen das ökonomische Nützlichkeits- und Erwerbsstreben oftmals wie eine lebensfeindliche Ethik der Selbstverleugnung erscheint. Die bürgerliche Rezeption Nietzsches äußert sich im Fin de Siècle deshalb nicht selten in der Aneignung einer 
dandyhaften, zugleich distanziert zynischen und verzweifelt egozentrischen, Attitüde, die auf elitäre Distinktion bedachtes äußeres Auftreten mit einer zurückgezogenen Innerlichkeit verbindet. Der Dandy ist es schließlich, der die nihilistische und passive Dekadenz, das negative „Dahinsterben des letzten Menschen“43, in die aktive und schaffende, sich im dionysischen Rausch vollziehende, Selbstzerstörung zu überführen versucht, ohne allerdings dieses Ziel zu erreichen. Er bleibt wie der Wahnsinnige (Nietzsche selbst) im modernen Vakuum zwischen Dekadenz und dionysischem Schöpferrausch stecken. ${ }^{44}$

Die Fluchtlinie, der Ausweg aus der Dekadenz der mediokren bürgerlichen Gesellschaft, artikuliert sich im Willen zu Distinktion und Vornehmheit 45 . Der Dandy steht dabei (nur) für einen Versuch (und Scheitern) dieser Flucht, die dem expandierenden und demokratisierenden, weil majorisierenden Effekt der Moderne, wie ihn Paul Valéry für Europa konstatiert hat, zum Opfer fällt: Das einzigartige Starke und Mächtige unterminiert im Zuge seiner Expansion, der Unterwerfung des zahlreichen Schwachen unter seine machtbegründenden Prinzipien, seine Einzigartigkeit und damit sich selbst.46 Der Wille zu Distinktion und Vornehmheit, der aus der entstehenden Massen- und Konsumgesellschaft des Fin de Siècle hervorgeht und sich in ihr ausbreitet, unterminiert sich ebenso in dem Maße, in dem das allgemeine Streben nach Distinktion und Vornehmheit repräsentativ und nivellierend wirkt. Wollen alle vornehm sein, wird die Vornehmheit ausgelöscht. Mithin geht der Dandy im Fin de Siècle daran zugrunde, dass er sich nicht mehr unterscheiden und hervorheben kann, da er allgemein geworden ist. ${ }^{47}$

Will man den Dandy und seine tatsächlichen diskursiven Praktiken der Selbstkonstitution im 19. Jahrhundert gleichsam formal literarisch charakterisieren, so drängt sich geradezu das Attribut aphoristisch auf. ${ }^{48}$ Denn offensichtlich besteht mehr als eine bloße Affinität des Dandys zur literarischen Form des Aphorismus. Die primäre kommunikative Ausdrucksform des Dandys waren aphoristische Sentenzen, die er eloquent in der Konversation platzierte.

43 Vgl. Deleuze: Nietzsche, S.189.

44 Zum pathologischen Niedergang des Dandys vgl. Moers: Dandy, S.303ff.

45 Vgl. Heller: Nietzsche, S.314.

46 Siehe Paul Valéry: Regards sur le monde actuel, Paris: Stock 1931, bes. S.49ff.

47 Vgl. Moers: Dandy; S.313f.

48 Vgl. auch Susanne Rossbach: Des Dandys Wort als Waffe. Dandyismus, narrative Vertextungsstrategien und Geschlechterdifferenz im Werk Jules Barbey d'Aurevillys, Tübingen: Niemeyer 2002. 
Mithin entsprach die Form des Aphorismus exakt dem Stil - als Medium der Transgression - des Dandys.

\section{Fazit: Der Dandy, Nietzsche und das Fin de Siècle}

In diesem Zusammenhang dandyhafter Selbststilisierung steht auch das diskursive Ereignis im Fin de Siècle, das sich mit dem Namen Friedrich Nietzsches verbindet. Nietzsche konstituierte nicht nur einen Diskurs ${ }^{49}$, sondern prägte nicht zuletzt in der Form des Aphorismus einen eigensinnigen ästhetisch inspirierten Denk-Stil aus, der wiederum gerade das Problem des Stils selbst - analog und in vielfältiger Beziehung zum Dandytum - zu einer ethisch-ästhetischen und eventuell politischen Frage moderner Subjektivierung erhob.

Georg Brandes charakterisierte bereits zeitgenössisch Nietzsches Denken 1890 als aristokratischen Radikalismus, der „in Aphorismen denkt“ und ,jedem Gedanken einen Hochdruck zu geben pflegt, der ihm eine paradoxale Physiognomie verleiht."50 Nietzsche definiert „großen Stil“ durchaus ganz im Sinne des Dandys als Pathos der Oberfläche ${ }^{51}$ und getreu seinem auf die antiken Griechen bezogenem Diktum, „oberflächlich aus Tiefe“ zu sein.52 In diesem Ästhetizismus des Stils, der sich vor allem mit Nietzsche verbindet, zeigt sich das Dispositiv Ethik/Ästhetik, und zwar im Sinne eines dandyhaften Lebens- und Denk-Stils:

Hinter dem radikalen ästhetischen Gestus verbirgt sich nämlich der ethische Affekt eines Aristokratismus, der zurückgeht auf die französische Pathosformel des 17. Jahrhunderts: der Affekt einer heroischen Anstrengung, den Schmerz ertragen zu können, ihn nicht zum Schrei werden zu lassen. ${ }^{53}$

Der Wille zum Stil kulminierte im Fin de Siècle des 19. Jahrhunderts zu einer ästhetizistischen Kriegsmaschine ${ }^{54}$, die im polyvalen-

49 Siehe hierzu Michel Foucault: „Was ist ein Autor?“, in: ders., Schriften, Bd.1, Frankfurt/Main: Suhrkamp 2001, S.1008ff.

50 Vgl. Georg Brandes: „Aristokratischer Radicalismus. Eine Abhandlung über Friedrich Nietzsche“, in: Deutsche Rundschau, Bd.LXIII, 1890; S.67.

51 Vgl. Karl Heinz Bohrer: Großer Stil. Form und Formlosigkeit in der Moderne, München: Hanser 2007; S.49f.

$52 \mathrm{Vgl}$. Nietzsche: Die fröhliche Wissenschaft, Vorwort zur zweiten Ausgabe, Leipzig 1887, Kritische Studienausgabe, Bd.3, hrsg. v. Giorgio Colli und Mazzino Montinari, S.352.

53 Bohrer: Großer Stil; S. 50.

54 Siehe zum Theorem der Kriegsmaschine Gilles Deleuze/Félix Guattari: Mille plateaux: Les Editions de Minuit, Paris 1980. 
ten Zeichen der décadence eine dezidiert ästhetische Umwertung der Werte konstituierte. „Denn nur als ästhetisches Phänomen ist die Welt und das Dasein ewig gerechtfertigt“, wie Nietzsche bereits 1870 in „Die Geburt der Tragödie“ postulierte. Hiermit verband sich nicht zuletzt ein emphatisch ästhetischer Rekurs auf das neue Kollektivsymbol „Leben“, das vor allem im zeitgenössischen Diskurs der Degeneration biopolitisch omnipräsent war und geradezu zum Konstituens einer korrespondierenden biopolitischen Gouvernementalisierung der Ethik im Zeichen gesellschaftlicher (nationaler) Selbstbehauptung, mithin eines hypostasierten Willens zur Macht, wurde. Damit avancierte „Leben“ eben auch zu einer genuin ästhetischen Perspektive der Beobachtung, unterschieden vom zeitgenössischen Diskurs der Degeneration, der „Leben“ vor allem als ethische Kategorie, ja geradezu als kategorischen Imperativ der Beobachtung konstituierte. Geschmack, Instinkt, Distinktion, Pathos der Distanz und Vornehmheit bilden die Modalitäten dieser antipolitischen Haltung.

Die korrespondierende Selbstbeschreibung und Selbstbehauptung als anti-politisch, die sowohl den Dandy des ausgehenden 19. Jahrhunderts als auch Nietzsche auszeichnete, konstituierte eine ästhetische Umwertung der Werte, die im kulturkritischen historischen Kontext des Fin de Siècle eventuell eine extrem hermetische ästhetizistische Stilistik initiierte. Und dies umso mehr, als Stil im korrespondierenden Dispositiv zum ästhetischen Medium par excellence der Selbstkonstitution avancierte. In diesem Sinne haben der Dandy und Nietzsche im Fin de Siècle den (Willen zum) Stil gleichsam diagonal auf einer ästhetizistischen Fluchtlinie der modernen Gesellschaft eingezeichnet. Der Stil wurde dabei zum Imperativ und Vehikel der Selbstkonstitution, und zwar in der Form dezidiert ästhetischer Wertsetzungen statt ethischer oder gar politischer Wertorientierungen.

\section{Literatur}

Aschheim, Steven E.: The Nietzsche Legacy in Germany 1890-1990, Berkeley: University of California Press 1992.

Barbey d'Aureville: Über das Dandytum und über George Brummell,

Berlin: Matthes \& Seitz 2006

Baudelaire, Charles: La Peintre de la Vie Moderne, in ders.: Oevres Complètes, Paris: Gallimard 1971.

Bergman, Peter: Nietzsche, „the last Antipolitical German“, Indiana: Indiana University Press 1987.

Bohrer, Karl Heinz: Großer Stil. Form und Formlosigkeit in der Moderne, München: Hanser 2007. 
Bohrer, Karl-Heinz: Die Ästhetik des Schreckens. Die pessimistische Romantik und Ernst Jüngers Frühwerk, München: Hanser 1978.

Brandes, Georg: „Aristokratischer Radicalismus. Eine Abhandlung über Friedrich Nietzsche“, in: Deutsche Rundschau, Bd.LXIII, 1890.

Bürger, Peter: Theorie der Avantgarde, Frankfurt/Main: Suhrkamp 1974.

Deleuze, Gilles/Guattari, Félix: Anti-Ödipus. Kapitalismus und Schizophrenie I, Frankfurt/Main: Suhrkamp 1977.

Deleuze, Gilles/Guattari, Félix: Mille plateaux: Les Editions de Minuit, Paris 1980.

Deleuze, Gilles: Differenz und Wiederholung, München: Fink 1992.

Deleuze, Gilles: Nietzsche et la philosophie, Paris: Presses Universitaires de France 2003 (orig. 1962).

Foucault, Michel: „Was ist ein Autor?“, in: ders., Schriften, Bd.1, Frankfurt/Main: Suhrkamp 2001.

Heller, Peter: „Nietzsche über die Vornehmen und die Vornehmheit“, in Peter Uwe Hohendahl und Paul Michael Lützeler (Hg.), Legitimationskrisen des deutschen Adels 1200-1900, Stuttgart: Metzler 1979.

Hörner, Fernand: Die Behauptung des Dandys. Eine Archäologie, Bielefeld: transcript 2008.

Lange, Wolfgang: „Im Zeichen der Dekadenz. Hofmannsthal und die Wiener Moderne“, in Rolf Grimminger (Hg.), Literarische Moderne. Europäische Literatur im 19. und 20. Jahrhundert, Reinbek: Rowohlt 1995.

Lange, Wolfgang: Der kalkulierte Wahnsinn. Innenansichten ästhetischer Moderne, Frankfurt/Main: Fischer 1992.

Moers, Ellen: The Dandy. From Brummel to Beerbohm, Lincoln (Nebraska): University of Nebraska Press 1978.

Nietzsche, Friedrich: Also sprach Zarathustra. Ein Buch für Alle und Keinen, Leipzig 1885, Kritische Studienausgabe, Bd. 4, herausgegeben von Giorgio Colli und Mazzino Montinari, Berlin: de Gruyter 1993.

Nietzsche, Friedrich: Die Geburt der Tragödie aus dem Geiste der Musik, Leipzig 1872, Kritische Studienausgabe, Bd. 1, herausgegeben von Giorgio Colli und Mazzino Montinari, Berlin: de Gruyter 1993.

Nietzsche, Friedrich: Götzendämmerung oder wie man mit dem Hammer philosophiert, Leipzig 1889, Kritische Studienausgabe, Bd. 6, herausgegeben von Giorgio Colli und Mazzino Montinari, Berlin: de Gruyter 1993.

Nietzsche, Friedrich: Jenseits von Gut und Böse. Vorspiel zu einer Philosophie der Zukunft, Leipzig 1886, Kritische Studien- 
ausgabe, Bd. 5, herausgegeben von Giorgio Colli und Mazzino Montinari, Berlin: de Gruyter 1993.

Nietzsche, Friedrich: Menschliches, Allzumenschliches. Ein Buch für freie Geister, Leipzig 1878, Kritische Studienausgabe, Bd. 2, herausgegeben von Giorgio Colli und Mazzino Montinari, Berlin: de Gruyter 1993, S.20.

Nietzsche, Friedrich: Zur Genealogie der Moral. Eine Streitschrift, Leipzig 1887, Kritische Studienausgabe, Bd. 5, herausgegeben von Giorgio Colli und Mazzino Montinari, Berlin: de Gruyter 1993.

Pine, Richard: The Dandy and the Herald. Manners, Mind and Morals from Brummel to Durrell, Basingstoke: MacMillan 1988.

Rossbach, Susanne: Des Dandys Wort als Waffe. Dandyismus, narrative Vertextungsstrategien und Geschlechterdifferenz im Werk Jules Barbey d'Aurevillys, Tübingen: Niemeyer 2002

Thomas, Richard Hinton: Nietzsche in German politics and society 1890-1918, Manchester: Manchester University Press 1983.

Valérie, Paul: Regards sur le monde actuel, Paris: Stock 1931. 
